\title{
Sex differences in human virtual water maze performance: Novel measures reveal the relative contribution of directional responding and spatial knowledge
}

\author{
Daniel G. Woolley ${ }^{\mathrm{a}, *}$, Ben Vermaercke ${ }^{\mathrm{b}, \mathrm{c}}$, Hans Op de Beeck ${ }^{\mathrm{b}, \mathrm{c}}$, Johan Wagemans ${ }^{\mathrm{b}}$, Ilse Gantois ${ }^{\mathrm{c}}$, \\ Rudi D'Hooge ${ }^{c}$, Stephan P. Swinnen ${ }^{a}$, Nicole Wenderoth ${ }^{\mathrm{a}}$ \\ a Department of Biomedical Kinesiology, Research Centre for Movement Control and Neuroplasticity, K.U.Leuven, Tervuurse Vest 101, 3001 Heverlee, Belgium \\ ${ }^{\mathrm{b}}$ Laboratory of Experimental Psychology, K.U.Leuven, Tiensestraat 102, 3000 Leuven, Belgium \\ ${ }^{\mathrm{c}}$ Laboratory of Biological Psychology, K.U.Leuven, Tiensestraat 102, 3000 Leuven, Belgium
}

\section{A R T I C L E I N F O}

\section{Article history:}

Received 13 October 2009

Received in revised form 7 December 2009

Accepted 12 December 2009

Available online 23 December 2009

\section{Keywords:}

Spatial navigation

Directional responding

Cue processing

Hippocampus

Parietal cortex

\begin{abstract}
A B S T R A C T
Sex differences in humans on virtual water maze navigation are well established when overall performance is measured, e.g., by the total time taken to find the hidden platform, total path length, or quadrant dwell time during probe trials. Currently, it is unknown whether males are better spatial learners than females, or if overall performance differences reflect other aspects of the task unrelated to spatial memory. Here, males and females were tested on a virtual analogue of the Morris water maze. We devised a novel method of analysis in which each trial was divided into an initial trajectory phase and search phase. We also implemented a new measure of spatial learning during early and late training, by including trials in which subjects were only required to indicate where they thought the hidden target zone was located. Consistent with previous reports, males outperformed females on overall measures of task performance. Males also performed significantly better on all initial trajectory phase variables. Interestingly, only small (non-significant) differences were observed during the search phase and when spatial learning was tested without the constraints of a typical water maze trial. Our results suggest that spatial knowledge regarding the location of the hidden target zone is not the main factor responsible for overall sex differences in virtual water maze performance. Instead, the largest sex differences were observed during the initial trajectory phase of the trial, which is thought to depend on effective processing of distal features of the environment.
\end{abstract}

(C) 2009 Elsevier B.V. All rights reserved.

\section{Introduction}

The Morris water maze is commonly used in rodent research to study the neurobiology of learning and memory (for a review, see [5]). The task requires subjects to learn the spatial position of an escape platform that is located in a circular pool of opaque water and hidden just beneath the surface. The importance and widespread use of the rodent version of the task has prompted an increasing number of researchers to investigate the performance of humans on analogous virtual versions $[1,2,11,18,19,22]$.

Previous studies in both rodents $[4,24]$ and humans $[1,2,18,19]$ suggest males perform better on the water maze compared to females. At present it is unclear which mechanisms underlie this sex difference. Are males better spatial learners, or is the advantage derived from other behavioural aspects of the task important to overall performance? Here, we examined which specific elements

\footnotetext{
* Corresponding author. Tel.: +32 16 329069; fax: +32 16329197.

E-mail address: daniel.woolley@faber.kuleuven.be (D.G. Woolley).
}

of behaviour comprising an individual trial lead to sex differences in human subjects.

In humans, it is well established that males outperform females on measures assessing overall virtual water maze performance. For example, Astur et al. [1,2] reported that adult males were faster in locating the hidden platform during training, a finding replicated in children [19]. These studies also found that when the platform was removed on a probe trial following training, males displayed a stronger search preference for the former platform quadrant. One potential mechanism mediating apparent sex differences on overall task performance in humans is the extent to which distal features of the environment can be used effectively. Two sources of distal information thought to be important to navigation in the water maze are (1) distal cues, defined as distinct visual features of the environment located outside the pool wall and (2) the geometry of the room in which the pool is located [22]. The pool wall itself and landmarks located within its boundaries can also be used, and are referred to as proximal cues. Sandstrom et al. [22] observed that males performed better than females when the geometry of the room was the only useful source of distal information available. Saucier et al. [23] demonstrated in a real-world navigation 
task that females preferred using landmark information, whereas males displayed a preference for cardinal directions (e.g. North, East) and distances. To determine which visual features in the environment were used during virtual water maze performance, Mueller et al. [18] recorded eye tracking data during the first $3 \mathrm{~s}$ of each trial. While both sexes directed gaze towards distal features of the environment significantly longer than the pool wall or search area, shorter trial latencies in males corresponded with a slightly longer focus on distal cues, and faster visual exploration of the environment early during training. These findings suggest that overall sex differences might arise from the ability of males to faster learn and discriminate important features of the distal environment.

Understanding precisely how distal and proximal cue processing influences behavioural water maze performance is receiving increased attention. While self-localisation within the room at the beginning of a water maze trial must be based on distal room cues, the initial trajectory towards a hidden platform can either be determined only by its spatial relationship to distal cues (place learning), or by combining distal and proximal information to select an initial trajectory towards the hidden platform based on its location relative to the pool wall (directional responding) [8-11]. Hamilton and colleagues argue that each trial begins with a directional response, followed by a local search for the platform controlled by judging distance to the pool wall. This idea is supported by a series of experiments that examined trajectories on probe trials in which the location of the pool was shifted within the room. In this situation, the absolute location of the learned platform position within the room is placed in competition with the relative location of the platform position within the pool. A trajectory towards the absolute location is consistent with place learning within the room, while a trajectory towards the relative location is consistent with a directional response based on proximal cues provided by the pool wall. They found that rats $[9,10]$ and humans [11] develop a preference to respond in the relative direction of the platform. Eye tracking data obtained from human subjects during performance of a virtual water maze showed that those subjects able to make direct trajectories to a hidden platform location directed their gaze towards distal cues early in the trial, before gradually shifting focus to the pool wall for the remainder of the trial [11]. In contrast, subjects classified as learning a non-direct strategy directed their gaze towards distal cues for a shorter duration early in the trial, before rapidly shifting focus to the pool wall. Although the authors indicated that a similar proportion of males and females were assigned to direct and non-direct trajectory groups, an analysis directly assessing sex differences was not reported.

The present study assessed if overall differences between human males and females on virtual Morris water maze performance could be attributed to specific components of the trial. New behavioural measures and analysis methods were developed in order to assess initial trajectory and local search performance independently. We hypothesised that males would perform better during initial direction selection, since this behaviour appears dependent on distal cues. It was an open question whether sex differences would be observed to a similar extent when accurate searching becomes more dependent on the pool wall.

\section{Methods}

\subsection{Subjects}

Twenty-two subjects ( 11 male, age range 18-24; 11 female, age range 18-26) participated in the study. All subjects had normal or corrected-to-normal vision and had not previously taken part in similar research. Prior to testing subjects were required to provide written informed consent to the procedures, which were approved by the Ethics Committee of the Katholieke Universiteit Leuven in accordance with the Declaration of Helsinki.

\subsection{Apparatus}

A custom virtual environment analogous to the Morris water maze (Fig. 1A) was constructed in Blender (www.blender.org) and rendered in MATLAB (v7.5, The Mathworks) by the Virtual Reality Toolbox (v4.6, The Mathworks). The environment consisted of a white circular tank (diameter $=16$ virtual reality units (vru), height $=0.5 \mathrm{vru}$ ) situated $0.5 \mathrm{vru}$ above ground level in the centre of a square room (length $=20 \mathrm{vru}$, height $=8 \mathrm{vru}$ ). The only distinguishing feature in the room was one filled blue circle (diameter $=0.5 \mathrm{vru}$ ) located on a wall approximately half way between the floor and the ceiling in the opposite corner of the room to the hidden target zone (Fig. 1A). Pilot testing indicated that the inclusion of a single distal landmark restricted the manner in which the task could be solved by non-spatial strategies such as view matching. Subjects viewed the room from a first-person perspective, and moved around via keyboard presses. Movement within the room was restricted to either forward displacement or orienting (i.e. rotating left and right in the same position). This enabled a subject's state to be classified during each trial as either stationary, moving forward or orienting. A single key press resulted in a forward movement of 0.1 vru or rotation of $1.5^{\circ}$. Data were recorded at $25 \mathrm{~Hz}$.

\subsection{Procedure}

Prior to the start of the experiment the virtual environment was described to subjects, who were instructed that their overall goal was to learn the precise location of a hidden target zone (the equivalent of a hidden escape platform) situated within the confines of the circular tank. Over the course of the experiment two types of trials were performed.

\subsubsection{Search trials}

The first type, referred to as a 'search trial', was similar to the standard rodent water maze trial. Each trial began from one of eight starting zones $\left(15^{\circ}\right.$ arc at $45^{\circ}$ intervals) located at the perimeter of the circular tank (Fig. 1D), with the exact position within a given starting zone varying from trial to trial. At the start of each trial subjects always faced the centre of the room, and then attempted to navigate to the hidden target zone as quickly and directly as possible. On trials in which the target zone was successfully intercepted, the walls of the room turned green and 'success' was displayed in the centre of the screen (Fig. 1B). Search trials had a maximum time limit that varied depending on the trial block (see Section 2.4). A strict time limit was imposed during training to encourage the learning of direct trajectories to the hidden target zone, and motivate fast performance. If a trial reached the maximum time limit the walls of the room turned red and 'time out' was displayed (Fig. 1C). Both the success and time out feedback periods lasted $3 \mathrm{~s}$, during which no forward movement or orienting was possible. At the end of the feedback period subjects were placed in a new starting zone and the next trial commenced immediately.

\subsubsection{Guess trials}

The purpose of 'guess' trials was to provide an estimate of spatial learning that was independent of strategies or time constraints which might have influenced performance on search trials. Instead of trying to intercept the target zone, subjects were required to move to where they thought the target zone was located and indicate this position via a key press. Subjects were instructed that the emphasis on guess trials was accuracy and not speed. No feedback was provided regarding the accuracy of each guess.

\subsection{Testing protocol}

The experiment was conducted in two sessions on consecutive days, each lasting approximately $60 \mathrm{~min}$. Testing consisted of the following stages: pre-training, early learning guess block, training, late learning guess block, and a visible target navigation test.

\subsubsection{Pre-training}

The first block of trials on day 1 consisted of pre-training. The purpose of pretraining was to enable all subjects to acquire limited knowledge regarding the general location of the hidden target zone, prior to the start of an intensive training protocol. The size of the hidden target zone in the pre-training block was relatively large (2.36 vru in diameter) and the maximum time limit relatively long ( $90 \mathrm{~s}$ ). Task difficulty with these parameters was low, and ensured subjects would not spend many trials searching for the hidden target zone without success. This was necessary, since pilot testing indicated that the more difficult version of the task used during training induced a high degree of variability between subjects in the number of trials required to first discover the location of the hidden target zone. In order to advance to the next stage, subjects first had to meet a pre-training criterion. Following three successful search trials, a guess trial was performed. Pre-training ended when the subject was able to provide an indication of the target location within 3 vru of the centre of the target zone. If the accuracy criterion was not met on the 

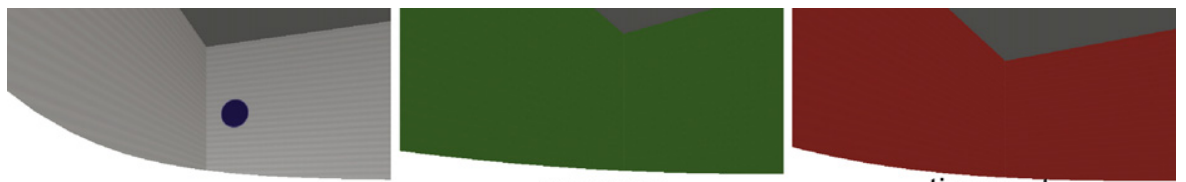

success

time out

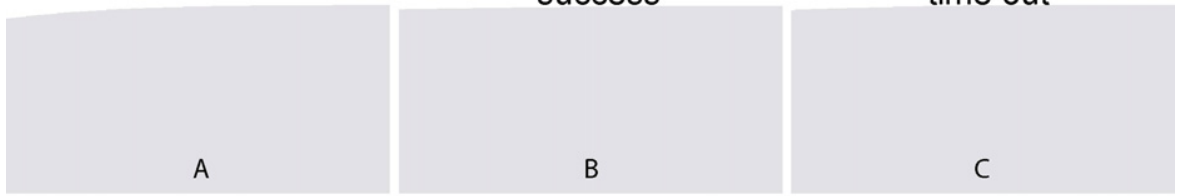

D

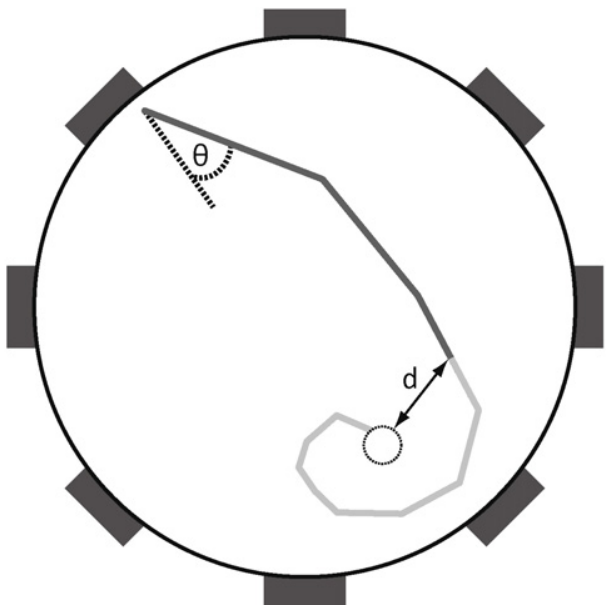

starting zone width

- initial trajectory phase

search phase

$\theta=$ initial heading error

$\mathrm{d}=$ initial trajectory

end point error

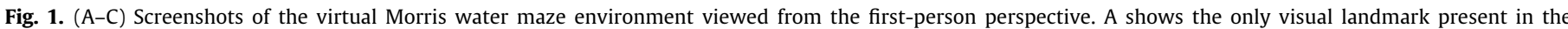
environment, the filled blue circle. Feedback at the end of successful and unsuccessful trials is shown in B and C respectively. (D) Example 'swim' path.

first guess trial attempted, another search trial was performed. From this point on in the pre-training block, every successful search trial was followed by a guess trial until the accuracy criterion was reached.

\subsubsection{Early learning guess block}

Once pre-training was complete, a block of eight guess trials were performed (one beginning from each of the eight starting zones). These trials provided a measure of spatial knowledge during early learning. Importantly, since no feedback was provided on guess trials, this measure could be obtained without influencing learning itself.

\subsubsection{Training}

Prior to the start of the main training paradigm, subjects were reminded that the location of the hidden target zone was fixed for the duration of testing. They were also informed that for the remaining search trials the hidden target zone was smaller ( $1.18 \mathrm{vru}$ in diameter) and that the maximum time limit on each trial was reduced to $35 \mathrm{~s}$. These parameters were modified in order to increase difficulty and enable task learning to be measured with higher sensitivity. In each training block, 16 consecutive search trials were performed. Four training blocks were completed on day 1 , and five training blocks on day 2 (total number of training block search trials $=144$ ).

\subsubsection{Late learning guess block}

Following the last training block on day 2, a second block of eight guess trials was performed, in order to provide a measure of spatial performance during late learning.

\subsubsection{Visible target navigation test}

At the end of the testing session on day 2, the final block of trials required navigation to a series of visible target zones (displayed as red platforms; diameter $=1.18 \mathrm{vru}$ ). These trials were conducted to assess navigation performance without a spatial memory component. Each trial began in the centre of the pool, with the subject immediately navigating to the first visible platform. Once the subject had intercepted the first platform location, it disappeared and a second platform appeared at a new location. Each trial involved continuous navigation to a sequence of eight visible platform locations, with subjects instructed to complete each trial as fast as possible. The sequence of platform locations was the same for all subjects, and five trials of the same sequence were completed.

\subsection{Behavioural measures}

Most conventional water maze measures, such as the time taken to find the platform or path length between the start position and the edge of the platform, assess overall performance. In the present study we evaluated overall task performance on search trials by calculating the time taken to find the hidden target zone (trial time), and the success rate within each block of 16 trials over the course of the training (success rate). With respect to the calculation of trial time, both successful and unsuccessful trials were included. In addition to the overall measures, we also examined if there were performance differences between males and females on more specific aspects of the task. Recent work by Hamilton et al. [8-11] suggests that directional responding is an important component of water maze performance. This aspect of behaviour was assessed by calculating the average angular distance between two vectors, the first defined by the start position and the hidden target zone position, the second by the start position and the subject position, over the first 1.5 vru of movement (initial heading error). Furthermore, each trial was divided into an initial trajectory phase and search phase (Fig. 1D). The initial trajectory phase was defined as the beginning of the trial until the distance travelled from the start position was equivalent to the distance between the start position and the nearest edge of the hidden target zone. The search phase was defined as the remainder of the trial. On $1.2 \%$ of trials the distance travelled from the start position was less than the distance from the start position to the nearest edge of the hidden target zone. On a further $27.8 \%$ of trials subjects made a direct trajectory to the hidden target zone. The composition of the subset of direct trajectory trials was $55.1 \%$ male and $44.9 \%$ female. For these cases, the initial trajectory component included the whole trial and no search phase was defined. Dividing the trial into trajectory and search phases was useful for calculating a number of new measures including: the distance from the end of the initial trajectory to the nearest edge of the hidden target zone (initial trajectory end point error) and the time taken to complete the initial trajectory (initial trajectory time). Spatial performance was evaluated during the search phase by calculating the average distance between the subject's position and the nearest edge of the hidden target zone at the original sampling rate of $25 \mathrm{~Hz}$ for the duration of the search phase (search proximity). All search trial measures which required calculating the distance to the hidden target zone used its nearest edge as the reference position, since the area within the hidden target zone could never be explored. Spatial knowledge was measured on guess trials before and after training, by calculating the distance between the centre of the hidden target zone and the 'guessed' position indicated by the subject (guess distance error). The centre of the hidden target zone was used to calculate guess distance 
error, since on guess trials it was possible to indicate a position within the area of the hidden target zone. Finally, performance on the visible target navigation task was assessed by calculating the time to acquire the sequence of eight visible platforms.

\subsection{Data analysis}

Differences between males and females during pre-training and on the visible target navigation test were assessed via independent $t$-tests. Performance during training was examined by way of a mixed model ANOVA with block as the within subjects factor (data averaged for each subject across 16 trials in each block, 9 blocks total) and sex as the between subjects factor. A mixed model ANOVA was also used to test for differences between early and late learning guess block performance (data averaged for each subject across 8 trials in each block, 2 blocks total). Partial eta-squared $\left(\eta^{2}\right)$ was used to report effect sizes for group main effects (see [21] for information about partial $\eta^{2}$ measures). The alpha level was set to 0.05 . We note that although the large number of statistical comparisons increased the chance of type I errors, multiple (non-independent) variables were used to describe similar aspects of performance.

\section{Results}

\subsection{Pre-training}

Males and females exhibited similar performance during pretraining. Independent $t$-tests did not reveal any significant differences in the number of search trials (mean $\pm S E$ : males $=4.6 \pm 0.3$, females $=5.3 \pm 0.6 ; p=0.36$ ) or guess trials (males $=1.4 \pm 0.2$, females $=2.2 \pm 1.8 ; p=0.50$ ) required to attain the pre-training accuracy criterion (guess distance error $<3$ vru). Guess distance error on the successful guess trial was not statistically different ( males $=1.5 \pm 0.3 \mathrm{vru}$, females $=1.9 \pm 0.2 \mathrm{vru} ; p=0.25$ ). Additional comparisons of trial time, initial heading error, initial trajectory end point error and initial trajectory time were all non-significant (range of $p$ values: $0.23-0.78$ ). The only statistically reliable difference between groups during pre-training was in search proximity, with females searching nearer to the hidden target zone than males (males $=7.3 \pm 0.2$, females $=6.5 \pm 0.2 ; p<0.01$ ). We caution that pre-training $t$-tests were conducted on a relatively small number of trials, and as such, too much weight should not be placed on these results. Nevertheless, these data provide a useful characterisation of performance upon initial exposure to the virtual water maze.

\subsection{Training}

\subsubsection{Overall task performance}

In accordance with previous studies, males exhibited better overall task performance during training, i.e. they found the platform faster and more often than females. Males achieved both a higher success rate (group main effect: $F(1,20)=10.6, p<0.01$, $\eta^{2}=0.35$; Fig. 2A) and shorter trial times (group main effect: $F(1,20)=19.0, p<0.001, \eta^{2}=0.49$; Fig. 2B). The absence of an interaction between group and block main effects for success rate $(F(8,160)=0.4, p=0.92)$ and trial time $(F(8,160)=0.3, p=0.97)$ indicates that males performed better on these measures in all training blocks. Overall task learning is represented by an increase in success rate (block main effect: $F(8,160)=10.6, p<0.001$ ) and reduction in trial time (block main effect: $F(8,160)=12.8$, $p<0.001$ ) over the course of training. Further analysis of trial time, which did not include timeout trials, revealed that even when considering only successful trials females were slower than males (males $=17.9 \pm 0.5 \mathrm{~s}$, females $=20.5 \pm 0.6 \mathrm{~s}$; group main effect: $\left.F(1,20)=11.8, p<0.01, \eta^{2}=0.37\right)$.

\subsubsection{Initial trajectory phase}

Males outperformed females on all initial trajectory phase variables during training. Males produced lower initial heading errors (group main effect: $F(1,20)=7.6, p<0.05, \eta^{2}=0.27$; Fig. $3 \mathrm{~A}$ ), lower
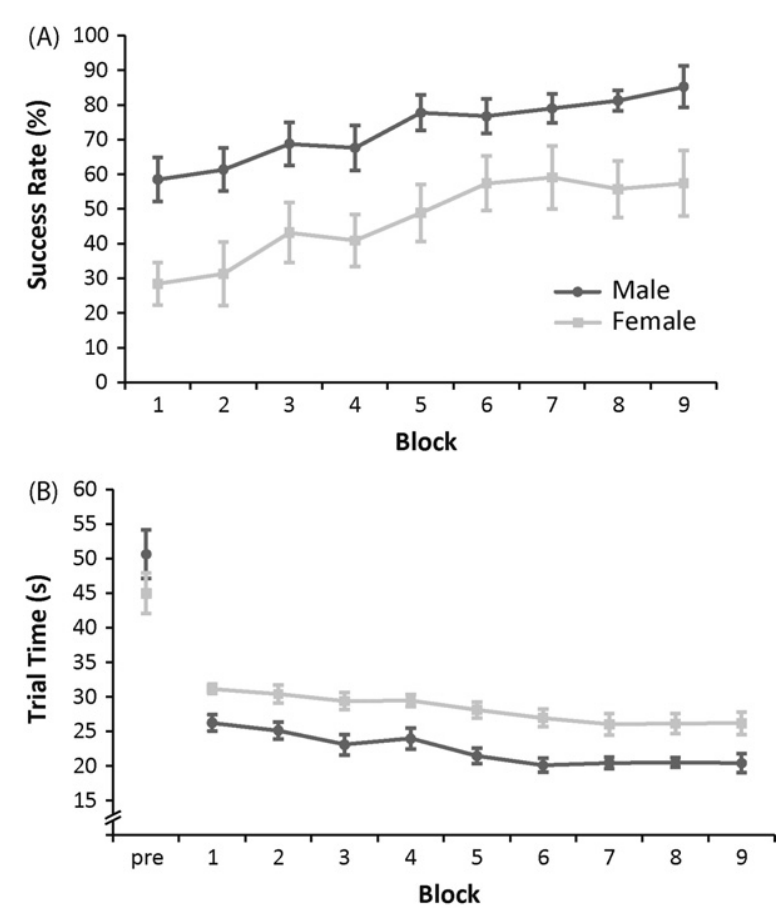

Fig. 2. Measures of overall performance (mean $\pm S E$ ). (A) Success rate in each training block displayed as a percentage. (B) Trial time for pre-training and training blocks.

initial trajectory end point errors (group main effect: $F(1,20)=10.8$, $p<0.05, \eta^{2}=0.35$; Fig. 3B), and faster initial trajectory times (group main effect: $F(1,20)=23.0, p<0.001, \eta^{2}=0.53$; Fig. $3 C$ ) compared to females. In other words, males were more accurate in selecting the direction of the hidden target zone at the start of the trial and made an initial trajectory that ended nearer the hidden target zone in a shorter time. A learning effect was found for all variables (block main effects, $F$ range: $4.8-16.4$, all $p$ values $<0.001$ ) with the better performance of males again apparent over all training blocks (non-significant interactions, $F$ range: $0.3-1.4$, all $p$ values $>0.22$ ).

\subsubsection{Search phase}

Search proximity revealed only small and non-significant differences between sexes. Based on the large reduction in search proximity between pre-training and training for both groups, it is evident that pre-training was successful in familiarising subjects with the general location of the hidden target zone. Moreover, although the search proximity was nearer to the hidden target zone for males in all training blocks, this difference was not statistically significant (group main effect: $F(1,20)=3.2, p=0.09, \eta^{2}=0.14$; Fig. 4A). A graphical representation of search proximity (Fig. 4B) shows focusing of the search area over the course of training. The relatively small advantage demonstrated by males during the search phase of a trial is in contrast with the much larger differences exhibited during the initial trajectory phase, and on overall task performance. This statistic was calculated from $71.0 \%$ of search trials in the training block, which were classified as having a search phase. The remainder of the training block trials were either direct trajectories to the hidden target zone (27.8\%), or the requisite distance from the starting position was not travelled in order for a search phase to be defined (1.2\%). Neither the block main effect $(F(8,160)=1.5, p=0.16)$ or interaction $(F(8,160)=1.1, p=0.35)$ were significant. 

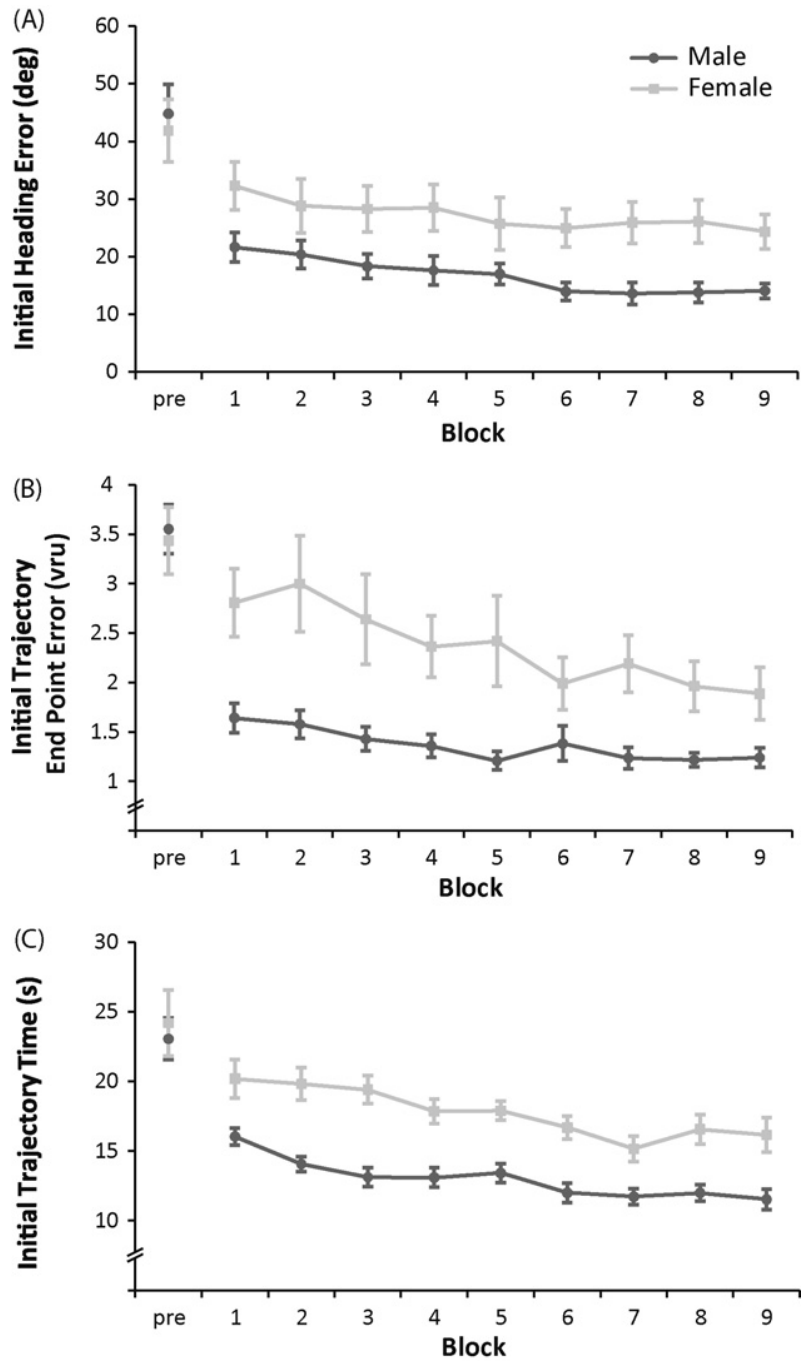

Fig. 3. Measures of initial trajectory phase performance for pre-training and training (mean $\pm S E$ ). (A) Angular error relative to the hidden target zone over the first 1.5 vru of movement. (B) Distance between the end point of the initial trajectory and the nearest edge of the hidden target zone. (C) Time taken to complete the initial trajectory phase of the trial.

\subsubsection{Guess trial blocks}

Spatial accuracy of early and late learning guess blocks was also not statistically different between groups (group main effect: $F(1,20)=2.5, p=0.13, \eta^{2}=0.11$; Fig. $5 \mathrm{~A}$ and $\left.\mathrm{B}\right)$, further suggesting that there was only a small and non-significant difference between males and females on the spatial component of the task. While similar guess trial performance was anticipated during the early learning guess block, since it was necessary for all subjects to meet the same guess distance error accuracy criterion during pre-training, significant sex differences were still not apparent following 2 days of search trial training (non-significant interaction: $F(1,20)=0.1, p=0.95$ ). It is important to note, however, that the average guess distance error of males was still slightly lower compared to females. As expected, spatial accuracy on guess trials was found to improve following training on blocks of search trials (block main effect: $F(1,20)=13.4, p<0.01)$.

\subsubsection{Visible target navigation}

Following the completion of water maze testing on day 2 , males were able to navigate to the sequence of visible target zones faster than females $($ males $=48.3 \pm 1.0 \mathrm{~s}$, females $=52.9 \pm 1.5 \mathrm{~s} ; p<0.05$ ).

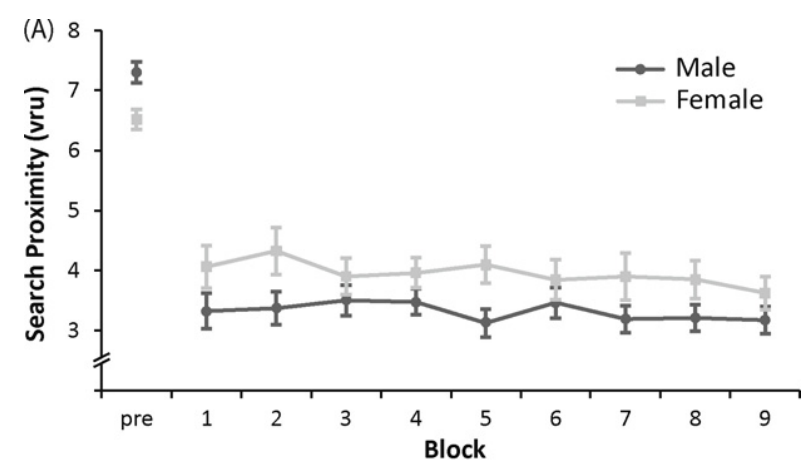

(B)

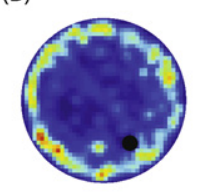

Male Pre

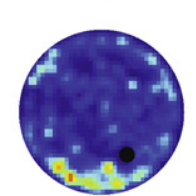

Female Pre

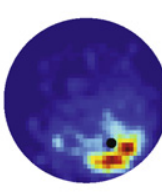

Male B1

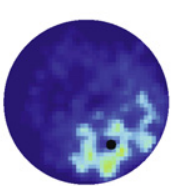

Female B1

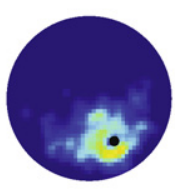

Male B5

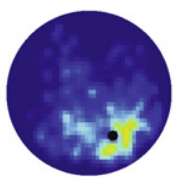

Female B5

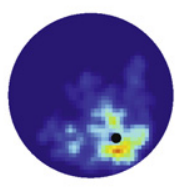

Male B9

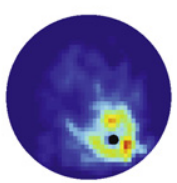

Female B9

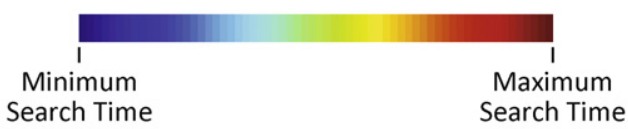

Fig. 4. Search phase performance during pre-training and training. (A) Average distance between the subject and hidden target zone during the search phase of the trial (mean $\pm S E$ ). (B) A graphical representation of changes in search proximity changes over the course of training. Group data is displayed. The black filled circle represents the location of the hidden target zone.

\section{Discussion}

Consistent with previous studies, males exhibited better overall Morris water maze performance than females. This was manifested as faster trial times and a higher success rate. Even when considering only successful trials, males found the hidden target zone faster than females. A novel finding of this study is that the difference in performance between sexes was greater during the initial trajectory phase of the trial compared to the search phase. Males displayed only a small (but non-significant) advantage when spatial learning of the hidden target zone was tested outside the context of a standard water maze trial. Interpretation of the extent to which differences were present on these measures is further supported by effect sizes. Measures specifically testing spatial knowledge of the hidden target zone resulted in the smallest effect sizes, while the largest were observed on initial trajectory phase variables. The similarity between sexes on measures specific to the acquisition of spatial knowledge in the present study is consistent with recent evidence from a real-world task where no sex differences were observed on spatial learning and memory performance [15].

\subsection{Large sex differences observed during the initial trajectory phase}

The largest water maze related sex differences in our paradigm were observed during the initial trajectory phase of a trial. Although initial heading error is not typically presented in either the human or rodent water maze literature, our result was consistent with a previous report that males were more accurate than females 


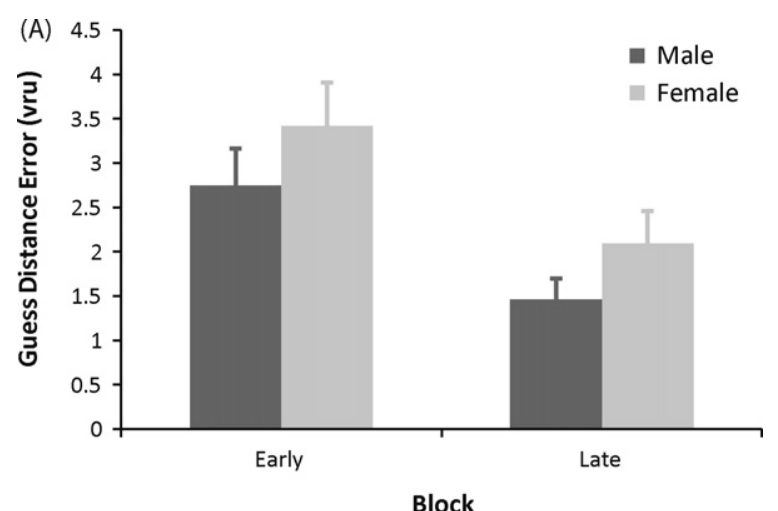

(B)
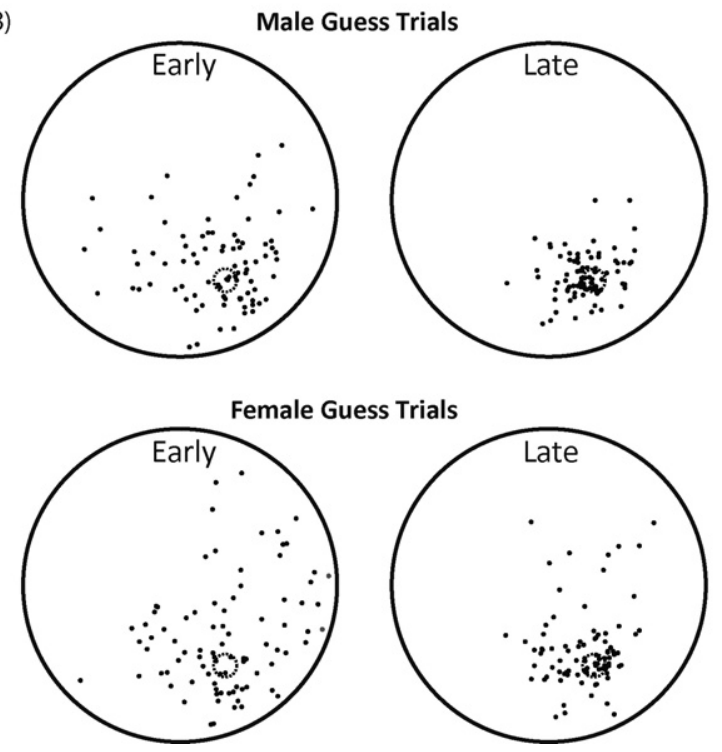

Fig. 5. Guess trial performance during early and late learning. (A) Distance between guess location and the centre of the hidden target zone (mean $\pm S E$ ). (B) Graphical representation of guess locations. Each black dot represents a single trial of an individual subject, with the data of all subjects displayed. The large circle indicates the pool wall, and the small circle the location of the hidden target zone.

in producing initial movement direction towards a hidden platform [1]. We also devised two novel measures, initial trajectory end point error and initial trajectory time, both of which confirmed a male advantage in accuracy and speed during this phase of the trial. Hamilton et al. [9-11] suggest that in order to produce an accurate initial trajectory towards the hidden target zone subjects must effectively integrate information obtained from both distal and proximal features of the environment. In our version of the task only one distal cue was present. While identification of this cue was critical for self-localisation within the environment, the relative sparsity of distal cues likely placed greater emphasis on the use of room geometry during initial direction selection. Our initial trajectory phase data support previous evidence that males are better able to use this type of distal information when performing navigation tasks $[18,22,23]$. The longer initial trajectory times for females were particularly problematic, since the maximum trial duration in our version of the task was only $35 \mathrm{~s}$ (during training). If the hidden target zone was not found immediately, females were left with less time to search for the hidden target zone. We suggest it was this aspect of the water maze, rather than the search phase, which contributed to the lower overall success rate of females.

Differences between males and females in initial trajectory phase performance, which relies on the effective processing of distal features in the environment, corresponds to reported sex dif- ferences in brain structures responsible for performing navigation tasks. An fMRI study, in which sex differences in behavioural navigation were observed, found that females activated right parietal and right prefrontal areas, whereas males were dependent on left hippocampus during navigation [7]. A lesion study in rats demonstrated that hippocampal and parietal regions are each responsible for processing different types of cues [25]. Hippocampal lesioned rats were able to learn a water maze in which only proximal cues were present within the pool, but showed a performance deficit on a distal cue version of the task. Rats with lesions in the associative parietal cortex showed the opposite pattern of behaviour. Thus, a female preference for a strategy dependent on parietal cortex and proximal cue processing might be the underlying reason why we observed a pronounced deficit during the initial trajectory phase, the part of the trial where the processing of distal features is most critical.

In addition to the lower accuracy and efficiency of females during the initial trajectory phase, clear sex differences were also observed on the visible target navigation task. At the end of the second day of water maze training, males navigated to a sequence of visible targets reliably faster than females. While the visible targets were presented in the same virtual environment as that used in the water maze, this test did not include a spatial learning component, or rely on the use of distal cues. Thus, it is likely that general aspects of interacting with a virtual environment unrelated to the water maze also contributed to overall sex differences. It is also possible that females are generally less responsive to virtual reality stimuli, evidenced by lower adaptation to visuovestibular conflicts during immersion in a virtual reality environment [26]. Males also show greater activation in the mesocorticolimbic system during computer game play, which implies a higher motivational state and greater sensitivity to reward [13]. Although we are unable to determine the relative contribution of non-water maze related factors and the initial trajectory phase to differences in overall task performance, it is probable that both played a role.

\subsection{Small sex differences observed on measures of spatial learning}

During the search phase, males demonstrated only a small (nonsignificant) advantage in search proximity. A similar variable was previously used by Gallagher et al. [6] to characterise probe trial behaviour in rodents, and was later shown by Maei et al. [16] to be a more sensitive indicator of spatial memory performance compared to common measures such as quadrant dwell time or the number of platform crossings. We adapted this variable to provide an indication of spatial learning during regular search trials by calculating the average distance to the hidden target zone over the period of the search phase. These data suggest that females were less impaired on this aspect of the task, which specifically reflects knowledge regarding the spatial location of the hidden target zone. Before and after training subjects also performed guess trials, a new type of virtual water maze trial designed to directly assess spatial learning of the hidden target zone outside the context of a regular search trial. The lack of time constraints on guess trials was particularly useful for revealing the true extent of spatial learning in the present study, since slow performance during the initial trajectory phase combined with short maximum trial durations resulted in limited overall success for females. Compatible with the search phase data, guess trials revealed only a small (nonsignificant) advantage to males in predicting the spatial location of the hidden target zone. It is important to note that the absence of a significant difference was despite the higher success rate of males during training, which resulted in more feedback regarding the precise location of the hidden target zone. Evidently, spatial 
knowledge of the hidden target zone location was not the main factor driving overall sex differences.

This result is in accordance with rodent studies that found sex differences on the water maze were not related to a spatial learning deficit per se, but are attributable to other aspects of the task. For example, evidence indicates that one of the major factors mediating sex differences in the rodent water maze is stress $[3,12,20]$. Swimming typically induces a higher stress response in female rodents $[14,17]$. Perrot-Sinal et al. [20] demonstrated that a pre-training procedure, which reduced stress related differences, enabled comparable performance on subsequent water maze training. Thus, it appears that in both humans and rodents there are aspects of the water maze task unrelated to spatial learning which contribute to overall performance differences between sexes.

\section{Conclusion}

Our results suggest overall sex differences in virtual Morris water maze performance can be attributed to the initial trajectory phase of the trial, where the processing of distal features within the environment is most critical. Smaller differences in performance were observed during the search phase, and on trials which tested spatial learning outside the context of a typical water maze trial. The division of each trial into initial trajectory and search phases, based on the work of Hamilton et al. [9-11], proved successful in revealing subtle differences in behaviour. Our results suggest that differences between sexes during virtual navigation tasks is not related to spatial learning per se, but appears to be the consequence of differences in the ability to effectively use specific types of distal information such as room geometry. It is worth noting that conventional water maze measures provide only an indication of overall performance, and are not sensitive to these aspects of the task. We devised novel measures and a new method of testing spatial learning in humans in order to examine the specific behaviours which comprise an individual water maze trial. Importantly, the initial trajectory and search phase variables reported here can be easily applied to rodent data. This method of analysis could prove to be a useful addition to future human and rodent studies, since they might capture the behavioural expression of two different mechanisms that both contribute to overall water maze performance but are mediated by different brain areas.

\section{Acknowledgments}

Funding for this project was provided by an interdisciplinary research grant from K.U.Leuven (IDO/06/004). We would like to thank Wim Claeys and Charlotte Vanheukelom for their assistance with data collection.

\section{References}

[1] Astur RS, Oritz ML, Sutherland RJ. A characterization of performance by men and women in a virtual Morris water task: a large and reliable sex difference. Behav Brain Res 1998;93:185-90.
[2] Astur RS, Tropp J, Sava S, Constable RT, Markus ET. Sex differences and correlations in a virtual Morris water task, a virtual radial arm maze, and mental rotation. Behav Brain Res 2004;151:103-15.

[3] Beiko J, Lander S, Hampson E, Boon F, Cain DP. Contribution of sex differences in the acute stress response to sex differences in water maze performance in the rat. Behav Brain Res 2004;151:239-53.

[4] Blokland A, Rutten K, Prickaerts J. Analysis of spatial orientation strategies of male and female Wistar rats in a Morris water escape task. Behav Brain Res 2006;171:216-24.

[5] D'Hooge R, De Deyn PP. Applications of the Morris water maze in the study of learning and memory. Brain Res Brain Res Rev 2001;36:60-90.

[6] Gallagher M, Burwell R, Burchinal M. Severity of spatial learning impairment in aging: development of a learning index for performance in the Morris water maze. Behav Neurosci 1993;107:618-26.

[7] Grön G, Wunderlich AP, Spitzer M, Tomczak R, Riepe MW. Brain activation during human navigation: gender-different neural networks as substrate of performance. Nat Neurosci 2000;3:404-8.

[8] Hamilton DA, Rosenfelt CS, Whishaw IQ. Sequential control of navigation by locale and taxon cues in the Morris water task. Behav Brain Res 2004;154:385-97.

[9] Hamilton DA, Akers KG, Weisend MP, Sutherland RJ. How do room and apparatus cues control navigation in the Morris water task? Evidence for distinct contributions to a movement vector. J Exp Psychol Anim B 2007;33:100-14.

[10] Hamilton DA, Akers KG, Johnson TE, Rice JP, Candelaria FT, Sutherland RJ, et al. The relative influence of place and direction in the Morris water task. J Exp Psychol Anim B 2008;34:31-53.

[11] Hamilton DA, Johnson TE, Readhead ES, Verney S. Control of rodent and human spatial navigation by room and apparatus cues. Behav Process 2009;81:154-69.

[12] Harris AP, D'Eath RB, Healy SD. Sex differences, or not, in spatial cognition in albino rats: acute stress is the key. Anim Behav 2008;76:1579-89.

[13] Hoeft F, Watson CL, Kesler SR, Bettinger KE, Reiss AL. Gender differences in the mesocorticolimbic system during computer game-play. J Psychiatr Res 2008;42:253-8.

[14] Kavaliers M, Galea LA. Sex differences in the expression and antagonism of swim stress-induced analgesia in deer mice vary with the breeding season. Pain 1995;63:327-34.

[15] Banta Lavenex P, Lavenex P. Spatial relational learning and memory abilities do not differ between men and women in a real-world, open-field environment Behav Brain Res 2010;207:125-37.

[16] Maei HR, Zaslavsky K, Teixeira CM, Frankalnd PW. What is the most sensitive measure of water maze probe test performance. Front Integr Neurosci 2009;3:4.

[17] Mogil JS, Sternberg WF, Kest B, Marek P, Liebeskind JC. Sex differences in the antagonism of swim stress-induced analgesia: effects of gonadectomy and estrogen replacement. Pain 1993;53:17-25.

[18] Mueller SC, Jackson CPT, Skelton RW. Sex differences in a virtual water maze: an eye tracking and pupillometry study. Behav Brain Res 2008;193:209-15.

[19] Newhouse P, Newhouse C, Astur RS. Sex differences in visual-spatial learning using a virtual water maze in pre-pubertal children. Behav Brain Res 2007;183:1-7.

[20] Perrot-Sinal TS, Kostenuik MA, Ossenkopp K, Kavaliers M. Sex differences in performance in the Morris water maze and the effects of initial nonstationary hidden platform training. Behav Neurosci 1996;110:1309-20.

[21] Pierce C, Block R, Aguinis H. Cautionary note on reporting eta-squared values from multifactor ANOVA designs. Educ Psychol Meas 2004;64:916-24.

[22] Sandstrom NJ, Kaufman J, Huettel SA. Males and females use different distal cues in a virtual environment navigation task. Cogn Brain Res 1998;6:35160.

[23] Saucier DM, Green SM, Leason J, MacFadden A, Bell S, Elias LJ. Are sex differences in navigation caused by sexually dimorphic strategies or by differences in the ability to use the strategies? Behav Neurosci 2002;116:403-10.

[24] Saucier DM, Shultz SR, Keller AJ, Cook CM, Binsted G. Sex differences in object location memory and spatial navigation in Long-Evans rats. Anim Cogn 2008;11:129-37.

[25] Save E, Poucet B. Involvement of the hippocampus and associative parietal cortex in the use of proximal and distal landmarks for navigation. Behav Brain Res 2000;109:195-206.

[26] Viaud-Delmon I, Ivanenko YP, Berthoz A, Jouvent R. Sex, lies and virtual reality. Nat Neurosci 1998;1:15-6. 